prokaryotes; therefore gut flora are not the source. A dietary source can be questioned, as it is unlikely that the Hsp110 would be recovered in a structurally intact form in the large intestine. And so the most likely source is the gut epithelium itself. Yet, Hsp110 does not possess a signal peptide and so cannot be secreted from IECs via the classical secretory pathway. Noncanonical secretion, as seen, for example, with FGF-2, is a possibility (14). However, this release pathway, and the analogous pathway in yeast, appear to transport very few proteins. Perhaps, then, Hsp110 is released into the intestinal lumen as a consequence of epithelial renewal, particularly in response to IEL activation (15). If this is the case, though, it might be expected that other abundant cytosolic chaperone proteins, such as Hsp90 and Hsp70, would be recovered in the intestinal contents as well. Nonetheless, perhaps inappropriately elevated levels of Hsp110 in the intestinal lumen serve as an immunological "trigger" leading to aberrant induction of CD1d expression, a subsequent activation of $\mathrm{NK} \mathrm{T}$ cells, and
IL-13-elicited destruction of IECs (Figure 1) (16). Whatever the mechanism, one is left to ponder the fascinating mystery of why IECs might (uniquely?) release Hsp110 into the gut lumen. Insights into the precise cellular source of the gut-lumen Hsp110 and the mechanism of its release will provide all-important clues to this new riddle in the everexpanding world of Hsp110 function.

1. Fiocchi, C. 1998. Inflammatory bowel disease: etiology and pathogenesis. Gastroenterology. 115:182-205

2. Blumberg, R.S. 2001. Characterization of CD1d in mucosal immune function: an immunotherapeutic target for inflammatory bowel disease. Keio J. Med. 50:39-44.

3. Mizoguchi, A., Mizoguchi, E., Takedatsu, H., Blumberg, R.S., and Bhan, A.K. 2002. Chronic intestinal inflammatory condition generates IL-10-producing regulatory B cell subset characterized by CD1d upregulation. Immunity. 16:219-230.

4. Page, M.J., et al. 2000. Cd1d-restricted cellular lysis by peripheral blood lymphocytes: relevance to the inflammatory bowel diseases. J. Surg. Res. 92:214-221.

5. Bendelac, A., et al. 1995. CD1 recognition by mouse NK1+ T lymphocytes. Science. 12:863-865.

6. Mendiratta, S.K., et al. 1997. CD1d1 mutant mice are deficient in natural $\mathrm{T}$ cells that promptly produce IL-4. Immunity. 6:467-477.

7. Colgan, S.P., Hershberg, R.M., Furuta, G.T., and Blumberg, R.S. 1999. Ligation of intestinal epithelial CD1d induces bioactive IL-10: critical role of the cytoplasmic tail in autocrine signaling. Proc. Natl.
Acad. Sci. U. S. A. 96:13938-13943.

8. Mowat, A.M., and Weiner, H.L. 1999. Oral tolerance: physiological basis and clinical applications. In Mucosal immunology. P.L. Ogra et al., editors. Academic Press Inc. San Diego, California, USA. 587-618.

9. Colgan, S.P., et al. 2003. Intestinal heat shock protein 110 regulates expression of CD1d on intestinal epithelial cells. J. Clin. Invest. 112:745-754. doi:10.1172/JCI200317241.

10. Lee-Yoon, D., Easton, D., Murawski, M., Burd, R. and Subjeck, J.R. 1995. Identification of a major subfamily of large hsp70-like proteins through the cloning of the mammalian $110-\mathrm{kDa}$ heat shock protein. J. Biol. Chem. 270:15725-15733.

11. Easton, D.P., Kaneko, Y., and Subjeck, J.R. 2000. The hsp110 and Grp1 70 stress proteins: newly recognized relatives of the Hsp70s. Cell Stress Chaperones. 5:276-290

12. Anderson, K.M., and Srivastava, P.K. 2000. Heat, heat shock, heat shock proteins and death: a central link in innate and adaptive immune responses. Immunol. Lett. 74:35-39.

13. Srivastava, P.K., and Amato, R.J. 2001. Hea shock proteins: the 'Swiss Army Knife' vaccines against cancers and infectious agents. Vaccine. 19:2590-2597.

14. Wakisaka, N., Murono, S., Yoshizaki, T. Furukawa, M., and Pagano, J.S. 2002. Epstein-Barr virus latent membrane protein 1 induces and causes release of fibroblast growth factor-2. Can cer Res. 62:6337-6344.

15. Guy-Grand, D., DiSanto, J.P., Henchoz, P., MalassisSeris, M., and Vassalli, P. 1998. Small bowel enteropathy: role of intraepithelial lymphocytes and of cytokines (IL-12, IFN-gamma, TNF) in the induc tion of epithelial cell death and renewal. Eur. J Immunol. 28:730-744.

16. Bouma, G., and Strober, W. 2003. The immunolog ical and genetic basis of inflammatory bowel disease. Nat. Rev. Immunol. 3:621-633.

\title{
Dendritic cells and the intestinal bacterial flora: a role for localized mucosal immune responses
}

\author{
Holm H. Uhlig and Fiona Powrie
}

Sir William Dunn School of Pathology, University of Oxford, Oxford, United Kingdom

Mammals coexist in an overall symbiotic relationship with a complex array of commensal bacterial flora that colonizes the gastrointestinal tract. These intestinal bacteria interface with cells of the mucosal immune system, including DCs (see the related article beginning on page 693). Here we discuss mechanisms of interaction between intestinal bacteria and DCs and the role of localized gastrointestinal immune responses.

J. Clin. Invest. 112:648-651 (2003). doi:10.1172/JCI200319545.

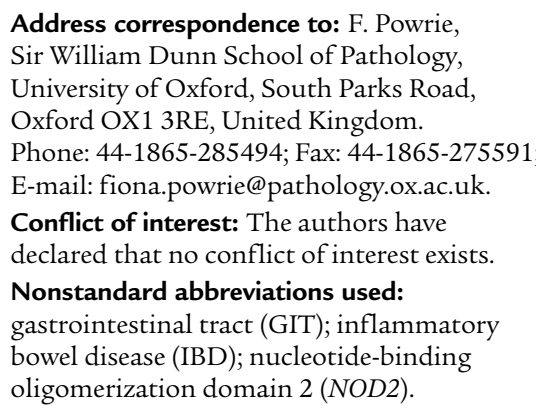

Inflammatory bowel disease is associated with a dysregulated immune response to intestinal bacterial flora

Through processes of evolutionary and individual adaptation, mammals coexist with an estimated 300 to 500 different species of commensal bacteria that colonize the gastrointestinal tract (GIT) in an overall symbiotic rela- tionship $(1,2)$. The presence of intestinal bacteria plays an important role in host metabolism, the development of the intestinal epithelium, and the intestinal immune system, and it also protects the host against rapid colonization by intestinal pathogens $(1,2)$. To allow sufficient defense against potential pathogens but restrict the immune response to nonpathogenic resident commensal bacteria, the mucosal immune system needs to be tightly regulated.

In human inflammatory bowel disease (IBD), which encompasses Crohn disease and ulcerative colitis, it is thought that a dysregulated $\mathrm{T}$ cell response to the intestinal bacterial microflora leads to chronic intestinal inflammation $(2,3)$. Although increasing evidence suggests that the intestinal flora is involved in the pathogenesis of human IBD, to date no specific bacterial pathogen has been identified. It seems more likely that different bacteria are involved in the initiation of the pathogenic immune response $(4,5)$. 


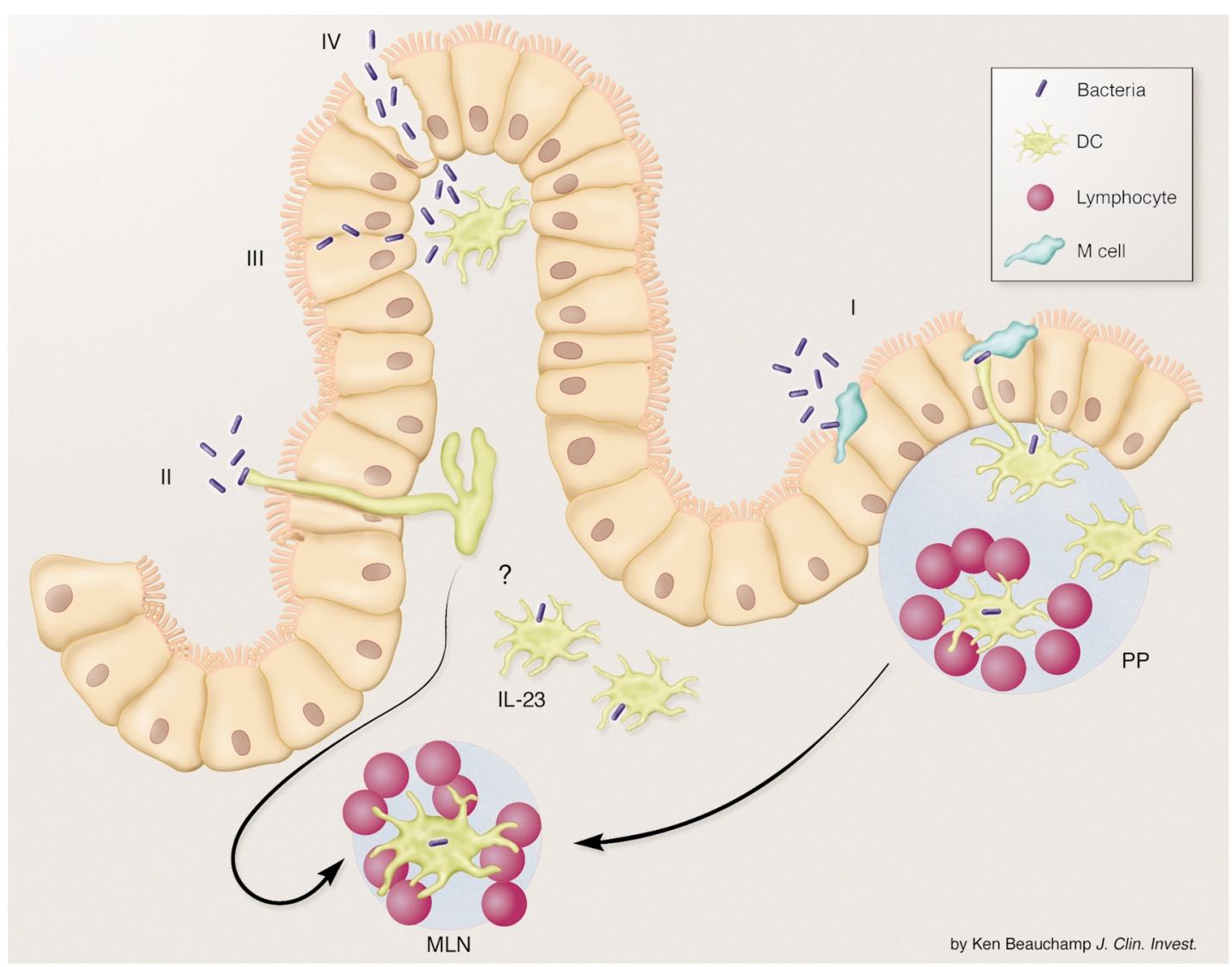

\section{Figure 1}

How do intestinal DCs sense the bacterial flora? Current theories of how DCs come into contact with the intestinal bacterial flora can be divided into those that propose an active sampling of bacteria by the host (I and II) and those that involve DCs acting as antigen-sampling cells when bacteria have already crossed the epithelial barrier (III and IV). M cells as specialized epithelial cells can mediate the uptake of bacteria toward DCs in the intestinal lymphoid tissue (I) (13). This mechanism can be exploited as an entry site by pathogens. In addition, it has been shown that DCs can reach through the basal membrane and the epithelial layer toward the lumen via dendrites (II) (21). In this case, DCs express the tight junction proteins occludin, claudin 1, and zonula occludens 1, by which they can keep the barrier integrity intact (21). DCs may also sample translocated bacteria that reach the lamina propria because of a low degree of physiological leakiness of the epithelium (III), and they might contribute to the clearance of pathogenic bacteria that reach the lamina propria via invasion and/or tissue damage (IV). Becker et al. describe a population of DCs in the crypt lamina propria of the terminal ileum that produce IL-23 and contain bacteria (9). Whether these DCs actively sample bacteria from the crypt lumen or respond to invasive bacteria is currently not understood. After antigen encounter, DCs travel in local lymphoid structures such as Peyer's patches (PP) and toward the draining mesenteric lymph nodes (MLN) to initiate or maintain $\mathrm{T}$ and $\mathrm{B}$ cell immune responses.

Recently it was found that a subset of patients with Crohn disease have loss-of-function mutations in the gene that encodes the nucleotidebinding oligomerization domain 2 (NOD2) protein (6). NOD2 is a pathogen-recognition receptor that recognizes muramyl dipeptide derived from bacterial peptidoglycans (7). Although it is not understood how mutations in the NOD2 pathway give rise to $I B D$, these findings suggest that alterations in the recognition of intestinal bacteria can contribute to chronic inflammation.

Studies of IBD models in rodents provide compelling evidence that the bacterial flora plays a key role in the pathogenesis of the disease, as chronic intestinal inflammation fails to develop under germfree conditions (8). However, precisely how commensal bacteria in the intestine interface locally with cells of the immune system to initiate and perpetuate intestinal inflammation remains unclear.

\section{Intestinal bacterial microflora induces localized IL-23 secretion by DCs}

The proinflammatory cytokine IL-12 has been implicated in the pathogenesis of IBD in a number of mouse models and also in the immunopathogen- esis of Crohn disease $(3,8)$. In order to study IL-12 production in situ under physiological conditions, Becker and coworkers (9) developed a transgenic mouse expressing a reporter gene under the control of the IL-12p40 subunit promoter (see their article in this issue of the JCI). Somewhat unexpectedly, they found that 40 transcription is restricted primarily to the terminal ileum, where it is produced by a subset of DCs. Recently it has become clear that $\mathrm{p} 40$ is a component not only of IL-12 (p40/p35) but also of the Th1inducing cytokine IL-23 (p40/p19) (10). Strikingly, the elevated IL-12p40 in the ileum, observed by Becker et al., 


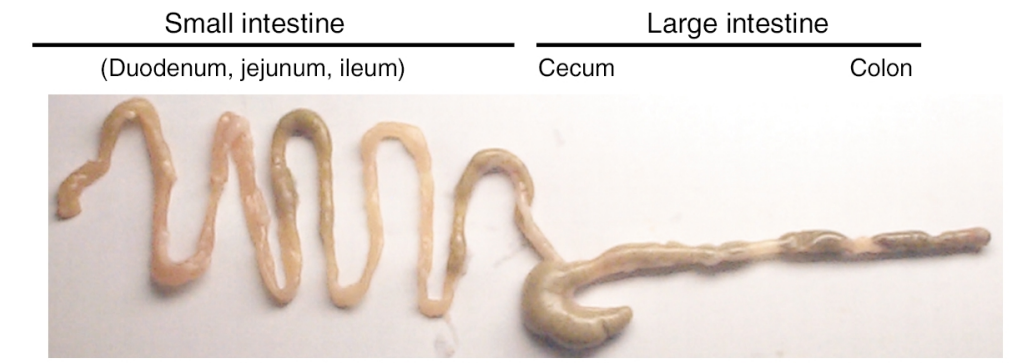

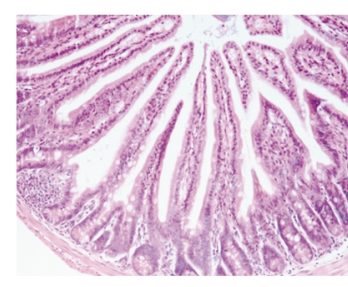

Small intestine

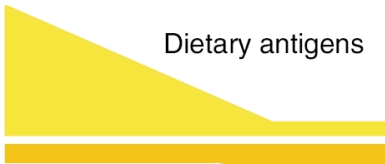

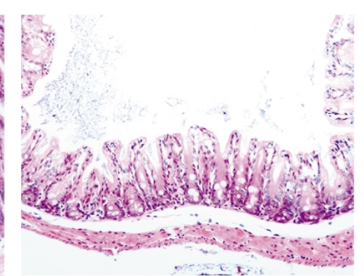

Cecum

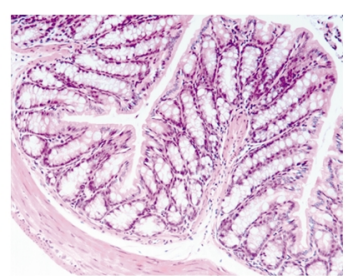

Colon
E. coli, Enterococcus spp., Lactobacillus spp. Proteus spp., Staphylococcus spp. Bacteroides spp., fusiform bacteria

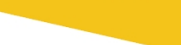

\section{Segmented filamentous}

bacteria

\section{Figure 2}

Specialized commensal bacteria occupy niches in different compartments of the GIT. The structure of the murine intestine in different parts of the GIT most certainly reflects its dominant purpose of digesting and absorbing nutrients. However, the presence and usage of various immune-response mechanisms also mirror the interplay with the respective resident intestinal bacteria in compartments that these bacteria are adapted to colonize as a permanent or temporary ecological niche. The immune response, in return, can shape the commensal bacterial flora (15). Whereas the total bacterial load increases toward the lower GIT, distinct bacteria are adapted to predominantly colonize niches in the small intestine or cecum (refs. 1, 14; and M. Strus et al., unpublished observations). Original magnification of the micrographs, $\times 200$. spp., species.

was accompanied by p19 but not p35 mRNA and increased IL-23 protein complex formation. IL-23 was produced in response to the bacterial flora, as it was not detectable in the ileum of mice housed under germfree conditions. Indeed, using an elegant combination of immunohistology and 16S-rRNA fluorescence in situ hybridization, the authors were able to show that some of the DCs with active p40 transcription contained intracellular nondegraded bacteria.

Restriction of DC IL-23 production to the terminal ileum and not the proximal small intestine or the colon suggests a specific activation state of CD $11 \mathrm{c}^{+}$cells in the former site. A key question is whether this represents the consequence of a hitherto unappreciated active sampling of commensal bacteria by DCs in the ileum, or is the result of selective colonization and invasion of a specific pathogen in the terminal ileum that leads to a local host immune response involving IL-23 secretion. The finding that bacteria-induced DC activation resulted in IL-23 as opposed to IL-12 production raises the possibility that IL-23 is an important mediator in intestinal inflammation, as has recently been reported in a model of cerebral inflammation (11). IL-23 may also play an important role in host defense against invading bacteria as a consequence of its ability to maintain Th1 cell memory responses by acting directly on CD4 ${ }^{+}$memory lymphocytes (10). Furthermore, IL-23 can activate DCs and macrophages via autocrine mechanisms (12). However, understanding of the biology of IL-23 is at an early stage, and further work is required to elucidate its role in host defense and intestinal inflammation.

The results of the study reported by Becker et al. (9) raise the question of how intestinal DCs come into contact with bacterial antigens. Several routes have been proposed, and these may vary depending on the nature of the bacterium (Figure 1) (13). Obviously, in order to prevent systemic infection, there is a clear need for the immune system to respond to bacteria that translocate from the lumen toward the lamina propria. Evidence also suggests that there are more active modes of antigen sampling involving $M$ cells or intra- and transepithelial DCs. However, the reason why the immune system bothers to actively sample intestinal antigens from the lumen is less clear. Such a mechanism might aid sustained antigen delivery for $\mathrm{T}$ cell-dependent and $T$ cell-independent IgA production (14). Alternatively, the active sampling of low amounts of intestinal antigens may be involved in inducing and maintaining $\mathrm{T}$ cell tolerance toward the intestinal bacterial flora.

\section{Localized mucosal immune responses as a result of differential colonization and pathogenicity}

It is tempting to speculate, as do Becker et al. (9), that the distinct elevation of IL-23 expression in the ileum reflects an increased susceptibility to inflammation in the terminal ileum, a site that is affected in patients with Crohn disease (3). However, this simple model with a dominant IL-23 response in the terminal ileum is difficult to reconcile with the finding that most mouse models of intestinal inflammation express dominant pathology in the cecum and colon, but not in the terminal ileum (8). 
The GIT provides distinct niches for colonization of commensal bacteria, as indicated by qualitative and quantitative differences in the bacterial flora throughout the GIT (Figure 2) $(1,15)$. The anatomical architecture in different parts of the GIT reflects its functional role in digestion and also in host defense (16). Thus, there are clear differences not only in the epithelial intestinal architecture, but also in the density of cells that are closely associated with the innate and adaptive immune response, such as goblet-producing cells, Paneth cells, DCs, and B and $\mathrm{T}$ lymphocytes. Furthermore, regional differences are found in the presence and density of mucosal lymphoid structures such as Peyer's or cecal patches, or isolated lymphoid follicles (16). It is likely that the lymphoid tissue throughout the GIT plays an important role in localized immune responses to bacteria that populate the respective compartment. Indeed, the development of the localized mucosa-associated immune system is only in part genetically determined; it is also functionally dependent on the presence of the bacterial microflora (17). In addition, differential complementary determining region-3 $\mathrm{T}$ cell receptor usage among $T$ cells in different regions of the colon provides further support for localized $\mathrm{T}$ cell immune responses within the large intestine (18). Interestingly, in HLA-B27 transgenic rats, the bacterial load in the cecum determines the severity of mucosal inflammation, and the development of colitis in $\mathrm{T}$ cell receptor- $\alpha$ mutant mice can be prevented by removal of parts of the cecum. These results suggest that the manipulation of bacteria and/or corresponding mucosa-associated lymphoid tissue in specific intestinal compartments can influence the induction of intestinal immunopathology (19, 20). Localized innate and adaptive mucosal immune responses may provide an efficient response to the respective flora but restrict extensive immunopathology. In this regard, the IL-12p40 promoter transgenic mice produced by Becker and coworkers (9) will be an excellent tool to study the interaction between particular bacteria and the host immune system and how this influences the localization of the immune response.

A dilemma for those using model systems in mice to study interactions between the intestinal bacterial flora and the immune system is that, despite increasing control of host genetics, afforded by the use of inbred mice with defined genetic alterations, our knowledge about the composition and function of the corresponding intestinal bacterial flora is limited to just a few bacteria. Because of the great variety of housing conditions, there may be large variations within the bacterial flora that might influence the activation state of the immune system and the interpretation of experiments in different laboratories. The further identification of distinct intestinal commensal and pathogenic bacteria and their ability to colonize and invade distinct compartments of the GIT will afford a better understanding of the interplay among different bacteria and between bacteria and the host in physiology and disease.

\section{Acknowledgments}

Holm H. Uhlig is supported by the European Society of Clinical Microbiology and Infectious Diseases and the Deutsche Forschungsgemeinschaft. Fiona Powrie is supported by the Wellcome Trust.

1. Hooper, L.V., and Gordon, J.I. 2001. Commensal host-bacterial relationships in the gut. Science. 292:1115-1118.

2. Guarner, F., and Malagelada, J.R. 2003. Gut flora in health and disease. Lancet. 361:512-519.

3. Shanahan, F. 2002. Crohn's disease. Lancet. 359:62-69.
4. Swidsinski, A., et al. 2002. Mucosal flora in inflammatory bowel disease. Gastroenterology. 122:44-54.

5. Linskens, R.K., Huijsdens, X.W., Savelkoul, P.H., Vandenbroucke-Grauls, C.M., and Meuwissen, S.G. 2001. The bacterial flora in inflammatory bowel disease: current insights in pathogenesis and the influence of antibiotics and probiotics. Scand. J. Gastroenterol. Suppl. 234:29-40.

6. Ogura, Y., et al. 2001. A frameshift mutation in NOD2 associated with susceptibility to Crohn's disease. Nature. 411:603-606.

7. Inohara, N., et al. 2003. Host recognition of bacterial muramyl dipeptide mediated through NOD2. Implications for Crohn's disease. J. Biol. Chem. 278:5509-5512.

8. Strober, W., Fuss, I.J., and Blumberg, R.S. 2002 The immunology of mucosal models of inflammation. Annu. Rev. Immunol. 20:495-549.

9. Becker, C., et al. 2003. Constitutive $p 40$ promoter activity and IL-23 production in the terminal ileum mediated by dendritic cells. J. Clin. Invest. 112:693-706. doi:10.1172/JCI200317464.

10. Trinchieri, G. 2003. Interleukin-12 and the regulation of innate resistance and adaptive immunity. Nat. Rev. Immunol. 3:133-146.

11. Cua, D.J., et al. 2003. Interleukin-23 rather than interleukin-12 is the critical cytokine for autoimmune inflammation of the brain. Nature. 421:744-748.

12. Puccetti, P., Belladonna, M.L., and Grohmann, U. 2002. Effects of IL-12 and IL-23 on antigen-presenting cells at the interface between innate and adaptive immunity. Crit. Rev. Immunol. 22:373-390

13. Didierlaurent, A., Sirard, J.C., Kraehenbuhl, J.P., and Neutra, M.R. 2002. How the gut senses its content. Cell. Microbiol. 4:61-72.

14. Macpherson, A.J., et al. 2000. A primitive T cellindependent mechanism of intestinal mucosal $\operatorname{IgA}$ responses to commensal bacteria. Science. 288:2222-2226.

15. Jiang, H.Q., Bos, N.A., and Cebra, J.J. 2001. Timing, localization, and persistence of colonization by segmented filamentous bacteria in the neonatal mouse gut depend on immune status of mothers and pups. Infect. Immun. 69:3611-3617.

16. Mowat, A.M. 2003. Anatomical basis of tolerance and immunity to intestinal antigens. Nat. Rev. Immunol. 3:331-341.

17. Yamanaka, T., et al. 2003. Microbial colonization drives lymphocyte accumulation and differentiation in the follicle-associated epithelium of Peyer's patches. J. Immunol. 170:816-822.

18. May, E., et al. 2002. Regional variation of the alphabeta $\mathrm{T}$ cell repertoire in the colon of healthy individuals and patients with Crohn's disease. Hum. Immunol. 63:467-480.

19. Rath, H.C., et al. 1999. Varying cecal bacterial loads influences colitis and gastritis in HLA-B27 transgenic rats. Gastroenterology. 116:310-319.

20. Mizoguchi, A., Mizoguchi, E., Chiba, C., and Bhan, A.K. 1996. Role of appendix in the development of inflammatory bowel disease in TCRalpha mutant mice. J. Exp. Med. 184:707-715.

21. Rescigno, M., et al. 2001. Dendritic cells express tight junction proteins and penetrate gut epithelial monolayers to sample bacteria. Nat. Immunol. 2:361-367. 